\title{
The Computational Cosmic Brain
}

\author{
Idan Ginsburg $^{1 *}$, Manasvi Lingam ${ }^{1}$ and Merrill Butler III $^{2}$ \\ ${ }^{1}$ Institute for Theory and Computation, Harvard University, Cambridge MA 02138, USA \\ ${ }^{2}$ Chemical Physics Laboratory, Concordia University, Irvine, CA 92612, USA \\ Email: iginsburg@cfa.harvard.edu
}

\begin{abstract}
The cosmic web exhibits some striking similarities to that of an animal brain. Despite its tremendous size the cosmos is many orders of magnitude less efficient at computation than the brains residing in Earth's biosphere. This shows just how information-rich life truly is.
\end{abstract}

Keywords: astrobiology, cosmic web, information theory, neural network

What drives the evolution of life on Earth? Commonplace answers include natural selection, genetic drift, and mutation. Such phenomena, while undoubtedly crucial, fail to properly explain the complex hierarchy of life on Earth which runs the gamut from molecules within cells to the entire biosphere. One fairly recent and unifying idea is that life is driven by information $[1,2]$. The most important organ in the human body, the brain, is in essence an information processing machine. Information flows to and from electrically excitable cells known as "neurons". Neurons transmit information to one another via nerve fibers known as "axons". Information is conveyed to a special junction between neurons known as a "synapse" while the receiving end of a neuron is a feathery filament known as a "dendrite". Microscopy of brain tissues has facilitated the detailed visualization of the complex network that spans the brain. One noteworthy result is that the neural network of most animal brains displays an uncanny resemblance to the large scale structure of the Universe. This leads to the questions: If the Universe were a cosmic brain, what would be its information capacity? Furthermore, what does such knowledge tell us about life on Earth?

The "cosmic web" is the observable Universe on the grandest of scales. On such scales, typically measured in megaparsecs (Mpc; where $1 \mathrm{Mpc}=10^{6} \mathrm{pc}=3.08 \times 10^{22}$ meters), galaxies and clusters of galaxies loosely resemble neurons and are connected to each other by filaments of matter and energy, analogous to axons, synapses, and dendrites in a brain (see Figure 1). The human brain has around $N_{B} \sim 10^{11}$ neurons [3], with a storage capacity of $I_{B} \sim 10^{16}$ bits [4]. In bits per neuron (bpn) this translates to

$$
S_{B}=\frac{I_{B}}{N_{B}} \sim 10^{5} \mathrm{bpn} .
$$

The human brain has $\sim 10^{4}$ synapses per neuron [5] and therefore $\sim 10^{15}$ synapses. In our order-ofmagnitude estimate, we find the number of bits per synapse to be $10^{16} / 10^{15}=10$, which agrees remarkably well with [4], who determined that the actual value is 4.7 bits per synapse.

The network of the cosmic web is such that the smallest observable structures are formed from the gravitational instability of overdense regions. The smallest structures susceptible to gravitational collapse are comparable in mass to that of the Sun [6]. Thus, in our cosmic web analogy, we choose stars to serve as nodes. The total number of nodes in the cosmic web, $N_{C W}$, is $10^{11}$ galaxies $\times 10^{11}$ stars per galaxy $=10^{22}$. Therefore the information capacity of the cosmic web is

$$
I_{C W}=S_{B} \times 10^{22} \text { nodes }=10^{27} \text { bits. }
$$

Here, we have explicitly assumed that the fractal nature of the cosmic web is akin to that of the human brain. In doing so, we further suppose that the number of filaments per node in the cosmic web is also comparable to the number of synapses per neuron in the brain. In principle, sub-stellar objects could also be considered nodes. As an example, if equation (2) was recalculated by incorporating planets, then $I_{C W}$ increases by three orders of magnitude [7]. Consequently, it is possible to assume that every single particle in the Universe can act as a bit [8], in which case the information capacity catapults to $\sim 10^{90}$ bits. However, extrapolating this value, by way of analogy, to the memory capacity of a human brain is 




Figure 1. The cosmic web as a giant brain? The background in this figure depicts the baryonic density in a slice of the cosmic web that was generated during the TNG300 simulation of IllustrisTNG. The scale from left to right is nearly $370 \mathrm{Mpc}$. The observable Universe is believed to be isotropic and homogeneous, and is manifestly similar in appearance to the neural network of the brain. This parallel naturally raises questions, which this paper addresses, such as: If the cosmos were one giant brain, how does it compare with our own? (Background illustration used with permission.)

erroneous. The storage capacity of a living brain is determined by the number of neurons and synapses, not the number of atoms and other subatomic particles, whose numbers are many orders of magnitude larger.

We can see that the cosmic web, in essence the cosmos, has the computational capacity of $10^{11}$ modern humans. There are approximately $10^{10}$ people on Earth, thus it would take 10 human-filled Earths to have the same computational power as the cosmos. However, if we assume that equation (1) applies to most creatures with brains, then as a whole insects have far more computational power than the entire class Mammalia. The adult fruit fly, Drosophila melanogaster, has on the order of $10^{5}$ neurons [9]. There are approximately $10^{19}$ living insects on the Earth at any given moment [10], thus the total information capacity for the class Insecta is $10^{19}$ insects $\times 10^{5}$ neurons $\times 10^{5} \mathrm{bpn}=10^{29}$ bits. Consequently, viewed in their entirety, insects have by far the most computational potential of all life (endowed with neural systems) on Earth. It takes only one Earth-like planet, replete with merely $1 \%$ the total population of insects relative to the Earth, to equal the computational power of the observable Universe. Another consideration is the computational surface density, which we define as

$$
\Phi=\frac{I}{4 \pi r^{2}}
$$


where $I$ is the information capacity and $r$ the radius. Earth has a radius of roughly $6400 \mathrm{~km}$, whereas the radius of the observable Universe is $\sim 14000 \mathrm{Mpc}[11]$. Choosing $I_{\text {Earth }} \sim 10^{29}$ bits, the computational density of Earth's biosphere is $\sim 10^{14}$ bits per square meter. From (2) and (3), the cosmic information density is on the order of $10^{-28}$ bits per square meter. This corresponds to an amazing 42 orders of magnitude difference between our biosphere and that of the cosmic web, thus illustrating how informationrich life on Earth truly is.

Acknowledgments. We thank Irwin Shapiro for useful feedback. We thank Lars Hernquist for granting us permission to use the background in Figure 1. We also thank Dimitar Sasselov and Neta Bahcall for supportive comments. This work was sponsored in part by Harvard University, and the Institute for Theory and Computation.

\section{References}

1. Hoyle, F. and Wickramasinghe, N. C., "Biological Evolution", Astrophysics and Space Science, vol. 268, pp. 5-75, 1999.

2. Walker, S., "Origins of life: a problem for physics, a key issues review", Reports of progress in physics, vol. 80 , pp. 092601, 2017.

3. Herculano-Houzel, S., "The human brain in numbers: a linearly scaled-up primate brain", Frontiers in Human Neuroscience, vol. 3, pp. 31, 2009.

4. Bartol, T.M. and Bromer, C. and Kinney, J. and Chirillo, M.A. and Bourne, J.N. and Harris, K.M. and Sejnowski, T.J., "Nanoconnectomic upper bound on the variability of synaptic plasticity", eLife, vol. 4, pp. e10778, 2015.

5. Drachman, D., "Do we have brain to spare?", Neurology, vol. 65, pp. 2004-2005, 2005.

6. Bonnor, W. B, "Jeans' formula for gravitational instability", Monthly Notices of the Royal Astronomical Society, vol. 117, pp. 104-116, 1957.

7. Dai, X. and Guerras, E., "Probing Extragalactic Planets Using Quasar Microlensing", Astrophysical Journal Letters, vol. 853, pp. L27, 2018.

8. Lloyd, S., "Computational Capacity of the Universe", Physical Review Letters, vol. 88, pp. 237901, 2002.

9. Burne, T. and Scott, E. and van Swinderen, B. and Hilliard, M. and Reinhard, J. and Claudianos, C. and Eyles, D. and McGrath, J., "Big ideas for small brains: what can psychiatry learn from worms, flies, bees and fish?", Molecular Psychiatry, vol. 16, pp. 7-16, 2011.

10. Williams, C.B., "The range and pattern of insect abundance", American Naturalist, vol. 94, pp. 137-151, 1960.

11. Gott, III, J. R. and Jurić, M. and Schlegel, D. and Hoyle, F. and Vogeley, M. and Tegmark, M. and Bahcall, N. and Brinkmann, J., "A Map of the Universe", Astrophysical Journal, vol. 624, pp. 463-484, 2005. 